\title{
Aceptabilidad de los suplementos alimenticios del programa Oportunidades
}

\author{
Angel Zarco, Antrop, (I) Gerardo Mora, Maestrante en Antrop,, (I) \\ Blanca Pelcastre, Dra en Psicol, ${ }^{(2)}$ Mario Flores, MC, ${ }^{(3)}$ Mario Bronfman, Dr en Soc ${ }^{(4)}$
}

\section{Zarco A, Mora G, Pelcastre B, Flores M, Bronfman M. Aceptabilidad de los suplementos alimenticios del programa Oportunidades. Salud Publica Mex 2006;48:325-33I.}

\section{Resumen}

Objetivo. Identificar usos y prácticas culturales para evaluar la aceptabilidad de los suplementos alimenticios del programa Oportunidades. Material y métodos. Estudio etnográfico con entrevistas a profundidad (43) -madres de niños menores de cinco años y mujeres embarazadas y en periodo de lactancia (MEPL)-; informantes clave (9); grupos focales (8) y observación directa en cuatro comunidades del norte, centro, rur y sureste de México. Resultados. El suplemento se prepara en distintas formas. Se consumió más en forma líquida que en solución de consistencia espesa. En las primeras ingestas causa vómito, diarrea y náuseas, pero estos malestares se superan posteriormente. Debido a la preferencia por la forma líquida, el suplemento se combina con leche. Entre las MEPL la aceptación fue generalizada. Conclusiones. El suplemento tiende a sustituir a la leche como un alimento en la dieta diaria. Se recomiendan evaluaciones en regiones con prácticas alimenticias culturalmente diferentes y considerar su relación con otros componentes del programa.

Palabras clave: Programa Oportunidades; suplementos nutricios; pobreza; desnutrición; México

\section{Zarco A, Mora G, Pelcastre B, Flores M, Bronfman M. Acceptability of dietary supplements of the national Mexican program "Oportunidades".} Salud Publica Mex 2006;48:325-33I.

\begin{abstract}
Objective. To identify cultural beliefs and practices to evaluate the acceptability of dietary supplements of Oportunidades Program. Material and Methods. Ethnographic study with in-depth interviews $(n=43)$-mothers of children less than five years of age and pregnant and breasfeeding women (PBW)-, key informants $(n=9)$; focus groups $(n=8)$ and direct observation, in four communities from North, Central, South and Southwest of Mexico. Results. The supplement was prepared in several different ways. The liquid preparation was best accepted; generally the supplement was mixed with milk. Initially, the supplement caused nausea, vomiting and diarrhea, but these symptoms disappeared with continued consumption. Acceptance was highest among PBW. Conclusions. The supplement tends to replace milk as a food product in the daily diet. There is a necessity to develop culturally specific evaluations in regions with different food intake practices. The study results should be interpreted in the context of other program components, such as health care and education.
\end{abstract}

Key words: Oportunidades Program; dietary supplements; poverty; malnutrition; Mexico

(I) Dirección de Organización en Sistemas de Salud. Centro de Investigación en Sistemas de Salud. Instituto Nacional de Salud Pública (INSP), Cuernavaca, México.

(2) Dirección de Salud Comunitaria y Bienestar Social. Centro de Investigación en Sistemas de Salud, INSP.

(3) Candidato al Doctorado en Políticas Alimentarias y Nutrición Aplicada. Centro de Investigación en Nutrición y Salud, INSP.

(4) Representante de la Fundación Ford para México y Centroamérica.

Fecha de recibido: 8 de marzo de 2005 - Fecha de aceptado: 9 de febrero de 2006

Solicitud de sobretiros: Dra. Blanca Pelcastre Villafuerte. Departamento de diseños y modelos alternativos de atención, Centro de Investigación en Sistemas de Salud, Dirección de Salud Comunitaria y Bienestar Social, Instituto Nacional de Salud Pública. Av. Universidad 655, Col. Sta. María Ahuacatitlán. 62508 Cuernavaca Morelos, México.

Correo electrónico: bpelcast@correo.insp.mx 
E ntre 1990 y 1995 la población de México creció de 81 a 91 millones de habitantes. ${ }^{1,2}$ Durante este periodo, en 1993, había en México 40 millones de pobres, de los cuales 13 millones se encontraban en situación de extrema pobreza. ${ }^{3}$

Para mediados de 1999 había en promedio 97 millones de mexicanos, de los cuales 40 millones seguían viviendo en la pobreza y 26 millones en pobreza extrema,, 5 aunque algunos especialistas aseguran que los pobres ya eran mayoría. ${ }^{6}$ En siete años el número de pobres extremos se había duplicado.

Ante este aumento de la población en pobreza extrema, durante la segunda mitad de los noventa, algunos programas gubernamentales destinados a combatir la pobreza se dirigían a ciertos sectores "prioritarios" y microrregiones del país en los que la situación era aún más extrema. Las categorías tradicionales de medición de la pobreza empezaban a perder la capacidad de explicar la situación en la que se encontraban estos sectores de la población que, según el Banco Mundial, sobreviven con menos de un dólar al día en una línea cercana a la indigencia, sobre todo en el medio rural., ${ }^{7,8}$

En 2002 se dieron a conocer nuevas cifras sobre pobreza en México y se dijo que la cifra alcanzaba 53.7\% del total de la población; ${ }^{9}$ el Banco Mundial comunicaba $46.8 \%$ de pobreza extrema. ${ }^{7}$ Estos datos alarmantes revelan la profunda desigualdad social y económica que se vive en el país, situación que resulta más preocupante si se considera que en el tercer informe de gobierno el Presidente de la República declaró que la de México es la novena economía del mundo. ${ }^{10}$ Este es, en términos generales, el contexto en el que opera el Programa de Desarrollo Humano Oportunidades (Oportunidades), que en la actualidad llega a 4.2 millones de familias mexicanas.

El Programa de Desarrollo Humano Oportunidades aparece como un conjunto de acciones intersectoriales del gobierno federal que busca disminuir los índices de pobreza extrema en México. El programa incluye un componente educativo, uno de salud y uno enfocado a la nutrición. El componente alimentario cuenta con una línea de acción muy importante cuyos objetivos son mejorar el crecimiento infantil, disminuir la prevalencia de desnutrición y mejorar la toma de decisiones de nutrición en el hogar. Uno de los insumos con los que cuenta el programa es un suplemento dietético que contiene micronutrimentos esenciales para mejorar la nutrición de los grupos vulnerables: niños menores de cinco años y mujeres embarazadas y en periodo de lactancia.

La desnutrición en la población menor de 5 años constituye un problema de salud pública en México, ya que existe una prevalencia de talla baja para la edad de $17.8 \%$, mientras que $7.6 \%$ de los niños presenta peso bajo para la edad y $2.1 \%$ presenta peso bajo para la talla. Asimismo, se han identificado deficiencias de micronutrimentos y cifras del $27.2 \%$ de la población menor de esta edad con anemia. En mujeres en edad reproductiva la principal deficiencia que se observa es la de hierro, aunada a las de zinc y vitamina C. ${ }^{11}$ Estas deficiencias imponen una carga importante sobre la salud de mujeres y niños en el país.

En los programas de suplementación nutricional es necesario asegurar la aceptabilidad del producto, así como su impacto en la población. El presente trabajo es resultado de la evaluación cualitativa realizada por el Instituto Nacional de Salud Pública acerca de la aceptabilidad de los suplementos alimenticios entre la población beneficiaria de Oportunidades.

El objetivo del estudio se centró en identificar usos y prácticas culturales que intervienen en la aceptación o rechazo de los suplementos nutritivos del programa Oportunidades. En este sentido, se entiende por usos y prácticas culturales locales a un conjunto de conocimientos que varían y que tienen sus particularidades a partir del contexto en el cual se reproducen. El empleo del término dieta resulta de importancia para el presente trabajo, ya que la dieta es el conjunto acostumbrado de alimentos preparados y consumidos regularmente en una cultura específica. Las diferencias de los patrones de consumo entre individuos, unidades domésticas o comunidades indican el lugar social dentro de grupos sociales más incluyentes. La dieta también evidencia la transformación social y cultural debido a que grupos humanos localizados abandonan la tradición a favor de tendencias alimentarias globales y estilos de vida en relación con ellas. Es importante destacar que en culturas que emplean "categorías humorales"*,12 (como en algunas regiones de México) la aceptación de alimentos nuevos, sobre todo para bebés o niños, puede llevar en sí la combinación de esas categorías con nuevas nociones sobre lo "nutritivo" por parte de la comunidad que los adopta. No obstante, el sabor y el costo tienen mucho más impacto sobre la selección de alimentos que las cualidades simbólicas, lo que afecta el consumo de alimentos de los individuos que experimentan algún momento orgánico en particular (embarazo o enfermedad). ${ }^{13}$

\footnotetext{
* Se conforman de cuatro cualidades básicas: calor, frío, sequedad y humedad. Es un sistema de valores presente en culturas mestizas e indígenas, donde el modelo común de oposiciones está presente. La dicotomía frío-calor es la base de equilibrio y donde el exceso de estas cualidades condicionan desquilibrio. En lo particular se orientan hacia el cuidado de la salud, tipo y consumo de alimentos.
} 


\section{Material y métodos}

Se realizó un estudio cualitativo con entrevistas a profundidad, grupos focales y entrevistas a informantes clave como técnicas de levantamiento de información, enmarcadas en una aproximación de diagnóstico rápido -RAP por sus siglas en inglés- ${ }^{14}$

El estudio se realizó durante los meses de noviembre y diciembre de 2002 en cuatro comunidades beneficiarias del programa, cada una perteneciente a un estado del norte, centro, sur y sureste del país: Tamaulipas, Guanajuato, Chiapas y Veracruz. Se realizaron en total 43 entrevistas a profundidad a madres de niños menores de cinco años, mujeres embarazadas y en periodo de lactancia (MEPL) a quienes se dirige el programa; nueve entrevistas a informantes clave en las que se identificó, sobre todo, personal responsable de la operación del programa en la localidad; se realizaron ocho grupos focales con mujeres usuarias y MEPL para complementar la información de las entrevistas. También se llevó a cabo una práctica de observación directa en las comunidades. El cuadro I reseña estas características.

La información de las entrevistas y los grupos focales se analizó por medio del software para análisis cualitativo Atlas.ti v.4.2.

\section{Resultados}

\section{La población estudiada}

Las comunidades estudiadas en cada estado eran rurales. La edad promedio de las entrevistadas se ubicó entre 25 y 30 años y su principal ocupación fue el hogar, con trabajo eventual en el campo o como empleadas domésticas. La mayoría de las entrevistadas no contaba con seguridad social. En cuanto a las características de la unidad doméstica, predominó la familia nuclear, el número promedio de hijos por familia fue de tres y la escolaridad promedio de ambos cónyuges, de primaria incompleta. Las principales ocupaciones de los varones fueron la agricultura y la albañilería; se identificó como una experiencia común entre ellos la migración hacia el interior de la República o hacia Estados Unidos. En general, cuando las entrevistadas y sus parejas desempeñan trabajos remunerados lo hacen en los empleos más bajos y peor pagados de la escala laboral. La carencia de fuentes de empleo en las comunidades, sobre todo en las de Chiapas, Guanajuato y Veracruz, fue evidente; por esta razón, en la comunidad de Guanajuato $45 \%$ de la población masculina migra a Estados Unidos. ${ }^{15}$

Los productos que conforman la base dietética de las familias visitadas son tortillas de maíz (de harina

\begin{tabular}{|c|c|c|c|}
\hline \multicolumn{4}{|c|}{$\begin{array}{l}\text { Cuadro I } \\
\text { ÑA METOdOLÓGICA }\end{array}$} \\
\hline $\begin{array}{l}\text { Lugar donde se obtuvo } \\
\text { la información }\end{array}$ & $\begin{array}{l}\text { Número y tipo } \\
\text { de informantes* }\end{array}$ & $\begin{array}{l}\text { Técnicas de levantamiento } \\
\text { de información }\end{array}$ & $\begin{array}{l}\text { Características de } \\
\text { los grupos focales }\end{array}$ \\
\hline Güémez, Tamaulipas & $\begin{array}{l}\text { - } 13 \text { usuarias del programa (embarazadas, } \\
\text { mujeres lactantes, mujeres con hijos } \\
\text { menores de cinco años) } \\
\text { - Cuatro responsables del programa } \\
\text { en la localidad } \\
\text { - Una promotora del programa }\end{array}$ & $\begin{array}{l}\text { - Entrevistas a profundidad } \\
\text { - Grupos focales } \\
\text { - Observación directa } \\
\text { - Pláticas informales }\end{array}$ & $\begin{array}{l}\text { Se formaron dos grupos con mujeres lactantes, } \\
\text { embarazadas o con menores de cinco años } \\
\text { (con seis participantes en cada uno) }\end{array}$ \\
\hline $\begin{array}{l}\text { El Bajío Bonillas, } \\
\text { Guanajuato }\end{array}$ & $\begin{array}{l}\text { - } 12 \text { usuarias del programa (embarazadas, } \\
\text { mujeres lactantes, mujeres con hijos } \\
\text { menores de cinco años) } \\
\text { - Una vocal del programa en la localidad }\end{array}$ & $\begin{array}{l}\text { - Entrevistas a profundidad } \\
\text { - Grupos focales } \\
\text { - Observación directa } \\
\text { - Pláticas informales }\end{array}$ & $\begin{array}{l}\text { Se formaron dos grupos con mujeres lactantes, } \\
\text { embarazadas o con menores de cinco años } \\
\text { (con ocho participantes en cada uno) }\end{array}$ \\
\hline $\begin{array}{l}\text { El Ejido Pedernal, } \\
\text { San Cristóbal de las } \\
\text { Casas, Chiapas }\end{array}$ & $\begin{array}{l}\text { - Nueve usuarias del programa } \\
\text { (embarazadas, mujeres lactantes, mujeres } \\
\text { con hijos menores de cinco años) } \\
\text { - Dos responsables del programa en } \\
\text { la localidad }\end{array}$ & $\begin{array}{l}\text { - Entrevistas a profundidad } \\
\text { - Grupos focales } \\
\text { - Observación directa } \\
\text { - Pláticas informales }\end{array}$ & $\begin{array}{l}\text { Se formaron dos grupos con mujeres lactantes, } \\
\text { embarazadas o con menores de cinco años } \\
\text { (10 participantes en cada uno) }\end{array}$ \\
\hline Tres Zapotes, Veracruz & $\begin{array}{l}\text { - Nueve usuarias del programa } \\
\text { (embarazadas, mujeres lactantes, mujeres } \\
\text { con hijos menores de cinco años) } \\
\text { - Una responsable del programa en } \\
\text { la localidad }\end{array}$ & $\begin{array}{l}\text { - Entrevistas a profundidad } \\
\text { - Grupos focales } \\
\text { - Observación directa } \\
\text { - Pláticas informales }\end{array}$ & $\begin{array}{l}\text { Se formaron dos grupos con mujeres lactantes, } \\
\text { embarazadas o con menores de cinco años } \\
\text { (nueve participantes en uno y } 10 \text { en otro) }\end{array}$ \\
\hline \multicolumn{4}{|c|}{$\begin{array}{l}\text { * El criterio para seleccionar a las mujeres participantes fue que estuvieran recibiendo los suplementos nutricionales de Oportunidades. } \\
\text { El criterio para seleccionar a los informantes clave fue su vinculación con la operación del mismo en cada localidad. Generalmente quienes participan en } \\
\text { los comités como vocales o promotoras son mujeres de la misma localidad }\end{array}$} \\
\hline
\end{tabular}


en Tamaulipas, y en Guanajuato de maíz y harina), frijol, huevo, arroz, verduras y frutas en menor medida; en ocasiones la carne, en especial de pollo y cerdo. En la comunidad de Chiapas el maíz es una de las principales fuentes de alimentación. En esta comunidad, como en algunas familias de las otras comunidades visitadas, las entrevistadas coincidieron en señalar que en ocasiones tienen problemas para solventar la alimentación familiar debido a sus escasos recursos económicos.

\section{EI programa}

En agosto de 1997 entró en acción el Programa de Educación, Salud y Alimentación Progresa. En marzo de 2002 Progresa se convirtió en el Programa de Desarrollo Humano Oportunidades.

Las madres de niños menores de cinco años y las mujeres embarazadas y en periodo de lactancia que son beneficiarias del programa deben asistir cada mes a su Centro de Salud, donde reciben una consulta médica en la que se registra su peso y talla; asisten a una plática sobre salud familiar y nutrición, y se les entrega una cantidad promedio de seis sobres de suplementos alimenticios por beneficiaria.

En todas las comunidades estudiadas se cuenta con comités integrados por miembros de la comunidad, casi siempre mujeres, encargados de informar, vigilar y supervisar la adecuada distribución de los suplementos, así como de otros beneficios otorgados por el programa.

A partir de los testimonios de los informantes clave se identificaron problemas de falta de abasto de suplementos en las comunidades de Guanajuato y Chiapas hasta por periodos de dos meses. También se identificaron problemas de organización al interior de la comunidad en los cuatro estados visitados. Estos problemas tenían que ver con cuestiones de organización por parte de algunas promotoras del programa, comercialización de los productos e inconformidades por la petición de cooperaciones para solventar gastos de logística y movilización de miembros de los comités en las comunidades, de lo cual es muestra el siguiente testimonio:

[...] porque ya también sabemos que en los mercados, las combis de Tenexapa cerca de San Cristóbal, los sobres de papilla allá los están vendiendo en el mercado de San Cristóbal [...] y algunas comunidades lo están vendiendo a San Cristóbal en el mercado a peso el sobre $[. .$.

\section{Las madres de niños menores de cinco años}

Preparación del suplemento alimenticio. El suplemento para niños menores de cinco años es una sustancia en polvo que se mezcla con agua hasta obtener una consistencia espesa, como papilla. Se presenta en sabores de plátano, chocolate y vainilla. En las cuatro comunidades las madres utilizan sobre todo dos términos para referirse al suplemento: "papilla" y "leche".

Todas las entrevistadas empezaron a ofrecer a sus hijos el suplemento de la manera antes indicada, pero ellos no lo aceptaron. A la mayoría de los niños no le gusta el suplemento en forma de papilla, así que las madres buscaron formas alternativas de preparación.

Formas alternativas de preparación y consumo. Las mujeres optaron por diferentes combinaciones en la búsqueda de una preparación mejor aceptada, por lo que hoy en día el suplemento se prepara y consume de formas muy diversas. Así, el suplemento se combina en cada comunidad con alimentos y frutas propios de la región y, en ocasiones, su preparación depende del clima y las estaciones del año. De esta manera, en Tamaulipas el suplemento se consume con avena, en panecillos, atole en época de frío y a manera de "chongos" o "congeladas" en época de calor. En Guanajuato se combina con leche de vaca, con té de canela, atole, arroz con leche, cajeta y mezclado con maíz en polvo -a esta forma de preparación se le conoce como "pinole"-; en esta comunidad también es común que los niños consuman el suplemento en polvo directamente del sobre. En Veracruz también se consume con atole, con café, té de canela, arroz con leche, con cereal y en polvo. En Chiapas se combina con café, atole de arroz, té de canela y en ocasiones se acompaña con tortillas o galletas. Algunas de las formas de preparación antes mencionadas incluyen métodos de cocción, con los cuales el suplemento pierde algunas propiedades nutritivas.

[...] A veces también así lo hago, así, en agua hervida o si no así revuelta con café, si no con tortilla. Sí, así lo vamos cambiando, [el agua] hierve 20 minutos.

Consumo y distribución en la familia. Según los informantes clave entrevistados, al comienzo del programa muy pocos niños consumían el suplemento, pero con el paso del tiempo -y con nuevas alternativas de preparación y consumo- son cada vez más los que lo consumen y está más extendida su aceptación.

No obstante que los niños beneficiarios del programa eran los que más consumían los suplementos, otros miembros de la unidad doméstica también participaban en el consumo, en particular los hermanos, aunque en ocasiones se incluía a los abuelos en caso de familias extensas. Muy pocas entrevistadas manifestaron que su pareja consumiera el suplemento. En la comunidad chiapaneca la mayoría de los miembros de las familias entrevistadas consumía suplemento, de 
lo cual se deduce que el número de veces y las cantidades de consumo del niño beneficiario son menores.

Resultó un hecho común que las familias visitadas tuvieran un excedente de uno o dos sobres, situación que ocurría mes con mes. En ocasiones, estos excedentes se regalan a familiares o vecinos. A través de la comunidad se extiende una red para regalar e intercambiar suplementos. Si bien no se identificó un sabor preferido por los beneficiarios en ninguna de las cuatro comunidades, en algunas familias los niños beneficiarios tienen un sabor preferido, y cuando los sobres de ese sabor se acaban algunas madres intercambian sobres. Por estas redes los suplementos llegan a algunas familias muy pobres que no son beneficiarias del programa y a quienes se les acabaron antes del fin de mes, y al mismo tiempo es la forma en que los que no los consumen se deshacen de ellos.

En las cuatro comunidades se identificó la utilización de dichas redes para vender los suplementos. Algunas personas simplemente los tiran a la basura o se los dan de comer a los animales de granja, práctica más extendida al inicio del programa.

Valoración de la consistencia. En general, los niños no aceptan la consistencia semisólida del suplemento y sus madres buscan maneras alternativas de prepararlo. La más frecuente es en forma líquida, de "leche" y, de acuerdo con la edad del niño, se sirve en vaso o biberón. No obstante, esta preparación, aunque líquida, no tiene la misma consistencia de la leche, pues algunas madres comentaron que era necesario agrandar el orificio del biberón para que no se tapara. Es común que a esta preparación se agregue azúcar y frutas, por lo que adquiere más bien el cuerpo de un licuado o una malteada.

Complemento o sustituto de la alimentación. Predomina una percepción del suplemento como "leche", pues para la mayoría de las entrevistadas tiene apariencia de leche y sabe a leche, además de que se preparara y se consume como tal. La leche es un elemento importante en la dieta de los mexicanos. Forma parte de la canasta básica de alimentos y México ha sido durante muchos años, por lo menos en la última década, uno de los primeros importadores de leche en polvo en el mundo. ${ }^{16}$ Así que la leche se considera en general un elemento más de la dieta y el suplemento percibido como tal tiende a remplazarla.

-Ah sí, yo pienso que es un sustituto de la leche...

-[...] Pero y la demás gente, ¿usted qué cree, que la ve como un sustituto de la leche?.

-Pues sí. O sea, económicamente sí, porque si le da papilla ya no compran leche, o sea, sí, sí, yo [pienso que esto se da] por la necesidad que hay.
Otro factor que interviene para que la gente relacione el suplemento con la leche es el hecho de que otros programas de asistencia social, como Alimenta, distribuyen sobres de leche en polvo en las comunidades. El suplemento también viene en sobres y su presentación es en polvo, las madres lo preparan como leche y para ellas tiene más o menos el mismo sabor que ésta.

Lo anterior, además del factor económico que limita la compra de leche, determina que el suplemento se considere no un complemento de la alimentación sino un alimento por sí mismo y tiende, en la mayoría de los casos, a incorporarse a la dieta como sustituto de dicho producto. Entendido como un alimento "completo", se combina de diversas formas como se combinaría la leche: con arroz, cereal o frutas.

Varias entrevistadas coincidieron en señalar que el suplemento era una ayuda para ellas porque "a veces no hay para leche" y destinan para otras cosas el dinero que tenían para ese gasto; o bien, compran menos leche que la acostumbrada y combinan su consumo con el suplemento. El recibir suplementos se convierte en una estrategia para administrar de otra forma el gasto familiar. Pero muchas veces, sobre todo en las familias y comunidades más pobres donde el dinero es aún más escaso, el suplemento ocupa el lugar de la leche o la sustituye casi de manera permanente. En Guanajuato una madre les decía a sus hijos: "no importa si les gusta o no, es lo único que hay, así que cómanlo".

En la mayoría de las familias visitadas en Chiapas casi todos los miembros de éstas consumían el suplemento; al respecto comentaba un entrevistado: "[...] es un alimento y quita el hambre". Esto es así no obstante que en esta comunidad la leche no es un alimento de consumo culturalmente generalizado, a diferencia del café.

En las cuatro comunidades se identificaron algunos casos de niños que se alimentan sólo con suplemento, sobre todo aquellos que toman biberón. El suplemento constituía el único alimento durante el día -aunque lo consumían varias veces al día-, pues según sus madres es lo único que "les cae bien".

Si bien se identificó una tendencia general a que el suplemento sustituya a la leche en la dieta familiar, también se identificaron algunos casos, por ejemplo en Tamaulipas, en los que el suplemento sí desempeña su función como complemento de la alimentación diaria de los niños: sus madres lo incluyen como postre o entre comidas.

Aceptabilidad y efectos negativos. En las cuatro comunidades se comprobó que en ocasiones, durante los primeros días de su consumo, el suplemento provoca 
malestares gastrointestinales, diarreas o vómitos; una expresión popular muy común es que "les cae de peso". De manera habitual, estos malestares desaparecen después de los primeros días mientras el organismo del niño se adapta al suplemento y sus madres continúan su administración, pues el personal de salud, por lo general, alerta sobre estos posibles efectos pasajeros; sin embargo, algunas madres decidieron suspender definitivamente el consumo del suplemento al percibir dichos malestares. En algunos de estos casos la suspensión del consumo se debió a una recomendación médica.

No, me lo daban pero ¿sabe qué?, a mí me dio mucho vómito una vez que lo tomé y casi me desmayaba yo del vómito que me provocó, y le agarré asco, y nunca [más] la tomé, para qué la voy a engañar.

Aceptabilidad y percepción de cambios positivos. En general, la identificación de cambios positivos se asocia con el aumento de peso de los menores, mayor apetito, se "desarrollan" más -crecimiento-; otras percepciones comunes son que incrementa su energía, mejora su estado de ánimo, de salud y disminuye la frecuencia de enfermedades respiratorias.

\section{Las mujeres embarazadas y en periodo de lactancia}

Preparación del suplemento alimenticio y valoración de su consistencia. En principio, la forma en que se prepara el suplemento alimenticio para las mujeres embarazadas y en periodo de lactancia difiere mucho de la que se usa en menores de cinco años, sobre todo por su consistencia. El suplemento para las embarazadas y en periodo de lactancia, según las indicaciones oficiales, adquiere en su preparación una consistencia líquida, como de leche. Todas las entrevistadas manifestaron estar de acuerdo con esta consistencia.

Aceptabilidad y percepción de cambios positivos. En las cuatro comunidades se encontró un número muy reducido de mujeres embarazadas. En Tamaulipas y Guanajuato se reconoció un consumo poco entusiasta del suplemento, mientras que en Veracruz las mujeres en este estado lo consumían sólo una vez al día, por la mañana.

En el caso del suplemento para las MEPL no es común que su consumo se comparta con otros miembros de la familia.

Si bien no se encontró un consumo generalizado del suplemento entre las MEPL, la principal razón de su consumo es que son conscientes de los beneficios para ellas y sus bebés. En el caso de las MEPL, el suple- mento es más un complemento de su dieta diaria que sustituto de algún alimento.

Entre las embarazadas predominaron las percepciones acerca de que el suplemento aumenta su apetito y peso, y que ayuda al nacimiento de niños fuertes y a la formación del sistema óseo del bebé.

Según las mujeres en periodo de lactancia, el suplemento causa aumento de la producción y calidad de la leche materna, propicia el nacimiento de niños con un peso adecuado y un mejor desarrollo del bebé.

Aceptabilidad y efectos negativos. En las mujeres embarazadas son frecuentes las náuseas, no siempre en relación con el suplemento, sino con el embarazo en sí; sin embargo, algunas mujeres las relacionan en gran medida con el suplemento y suspenden su consumo.

Además de las náuseas, entre las MEPL se identificaron también diarreas. Estos malestares, igual que en el caso del suplemento para menores de cinco años, suelen desaparecer después de unos días, mientras el cuerpo se acostumbra al suplemento. No obstante, en algunos casos se suspendió el consumo de los suplementos cuando no era bien aceptado, ya sea por recomendación médica o por decisión propia, mientras que otras continuaron el consumo después que desaparecieron los malestares.

\section{Discusión}

No obstante que al inicio del programa los suplementos eran poco consumidos por la población, según los prestadores de servicios de salud entrevistados, en la actualidad esa tendencia cambió y el número de personas beneficiarias que los consume aumentó. Su aceptabilidad ha ido en aumento y ahora el suplemento es bien recibido por la mayoría de la población beneficiaria, según lo muestran estudios puntuales en varios estados de la República. ${ }^{17}$

Por otra parte, la consistencia y el sabor de los suplementos resultan ajenos a la dieta diaria de los niños beneficiarios en las comunidades visitadas; por lo tanto, las madres buscaron formas alternativas y muy variadas de preparación y consumo, de acuerdo con los usos y prácticas alimentarias locales. Una estrategia presente en los distintos contextos estudiados fue la combinación del suplemento con alimentos que forman parte de la dieta cotidiana.

El concepto de "complemento alimenticio" parece tener un lugar muy particular en el imaginario cultural alimentario de las comunidades que colaboraron en el presente estudio. El aspecto y el sabor de la papilla, más que propiciar su aceptación, evidencia su relación con algo ajeno a la dieta local, que sabe mal y cuyo consu- 
mo es obligatorio. El suplemento, entendido como un alimento en sí mismo y no como complemento, tiende a asociarse con la leche y a integrarse como tal en la dieta cotidiana de la población beneficiaria.

El éxito de los suplementos dietéticos en la mejora del estado nutricional de la población tiene relación directa con la aceptación y el nivel de consumo que logran en la diversidad de ámbitos socioculturales en que dicha población se encuentra inserta. El hecho de que un suplemento no cumpla su función de complemento alimenticio y en la práctica sustituya a un alimento de vital importancia en la dieta de la población a la que se dirige, como es la leche, constituye un obstáculo en la consecución de los objetivos trazados en el componente de Alimentación del programa Oportunidades, al mismo tiempo que modifica la organización cultural de la alimentación en las comunidades a las que se dirige.

Cambiar la presentación y la consistencia de los suplementos dirigidos a los menores de cinco años para que resulten más comunes para la gente podría mejorar su aceptación por parte de la población.

Se recomienda la elaboración de campañas informativas que incluyan pósteres, carteles y otros recursos didácticos donde se resuman los principales beneficios del consumo de suplementos para el niño y la mujer, con casos concretos como ejemplos. El papel de la madre resulta estratégico para mejorar la salud de los niños y otros miembros de la familia; en ellas deben centrarse los esfuerzos para transmitir la importancia de un adecuado consumo de los suplementos. De igual manera, resultaría benéfica la elaboración de pequeños folletos o manuales dirigidos al personal de salud de primer nivel, en los que se identifiquen los riesgos y problemas más comunes en el consumo de los suplementos, la importancia del seguimiento y un control riguroso de su consumo. Beneficiaría también incluir las instrucciones para el personal de salud, para que informen e instruyan a la gente acerca de la composición nutricia del suplemento, con el fin de evitar la proliferación de ideas erróneas en torno a él.

A la fecha, en el territorio nacional conviven alrededor de 56 grupos étnicos, cuyos orígenes culturales, costumbres, visiones del mundo y prácticas alimentarias difieren unas de otras. Evaluaciones de la aceptabilidad de los suplementos dietéticos de programas de asistencia social se han llevado a cabo previamente, ${ }^{18,19}$ sin embargo, han sido estudios realizados en una sola comunidad y en un solo estado del país.

Las evaluaciones de la aceptabilidad de los suplementos dietéticos que otorga Oportunidades y su aparente éxito ${ }^{20}$ no deben desligarse de su relación con el éxito obtenido por otros componentes del programa, sobre todo por el componente de salud, en el que se debe resolver la escasez de medicamentos y el acceso a servicios de salud de segundo y tercer nivel, y tampoco fuera de su relación con el avance en otros ámbitos importantes del combate a la pobreza, de manera concreta, en este caso, la generación de empleos y las limitadas oportunidades de educación media superior y superior para amplios sectores de la población.

\section{Referencias}

I. Instituto Nacional de Estadística, Geografía e Informática. XI Censo General de Población y Vivienda, 1990. Aguascalientes: INEGI, 1992.

2. Instituto Nacional de Estadística, Geografía e Informática. Conteo de Población y Vivienda, 1995. Aguascalientes: INEGI, 1997.

3. Alonso F. Producción y consumo de leche en el país y el impacto de las importaciones y exportaciones. Ponencia presentada en el Foro sobre Rastro, clandestinaje, contaminación y recría del ganado lechero, Memoria, Comisión de Ecología y Medio Ambiente, Comisión de Ganadería, 1998.

4. Instituto Nacional de Estadística, Geografía e Informática. XII Censo General de Población y Vivienda, 2000. Tabulados Básicos. Aguascalientes: INEGI, 200 I.

5. Consejo Nacional de Población. Disponible en: http:// www.conapo.gob.mx/.

6. Boltvinik J, Hernández E. Pobreza y distribución del ingreso en México. México: Siglo XXI, 1999.

7. González R. Uno de cada dos habitantes del campo en México vive en la indigencia: BM. La Jornada 30 de Julio 2002

8. Lustig N. La pobreza y el Programa Progresa. En Alivio a la pobreza. Análisis del Programa de Educación, Salud y Alimentación. Memoria del Seminario Progresa y Política Social, CIESAS, México, 1998.

9. Presidencia de la República. Actividades de la presidencia.

Oportunidades. Conexión MX - Charla con Rogelio Gómez-Hermosillo, Coordinador Nacional de Oportunidades. Enero de 2005, México. 10. Tercer Informe de Gobierno. Documento en internet. Disponible en http://tercer.informe.presidencia.gob.mx. Consultado: septiembre de 2003. II. Instituto Nacional de Salud Pública. Encuesta Nacional de Nutrición 1999. Estado nutricio de niños y mujeres en México; 2001.

12. Lozoya X, Zolla C. La medicina invisible. México: Folios Ediciones, 1986. 13. Barfield T. Diccionario de Antropología. México: Siglo XXI, 2000.

14. Scrimshaw S, Hurtado E. Procedimientos de Asesoría Rápida para programas de nutrición y atención primaria de salud. California: UNU, UNICEF, CEL, 1988.

15. Instituto Nacional de Estadística, Geografía e Informática. XII Censo General de Población y Vivienda. Sistema Municipal de Bases de datos (SIMBAD). Disponible en: http: //www.inegi.gob.mx

16. Del Valle MC, Álvarez AG. La producción de leche en México en la encrucijada de la crisis y los acuerdos del TLCAN. Ponencia presentada en la Reunión de LASA. Guadalajara, Jalisco: México, 1997.

17. Flores M, Safdie M, Sotres D, Rivera J. Evaluation of the acceptability and intake by women and children of nutritional supplements fortified with three forms of iron. Experimental Biology, 2003. Abstract No. LB37I. 18. Martínez Salgado H MC et al.Aceptabilidad a suplementos nutricios en mujeres embarazadas o lactando y niños menores de cinco años. Salud Publica Mex 1999;4:163-169.

19. Rosado JL, Rivera M, López G, Solano L, Rodríguez G, Casanueva E, et al. Desarrollo y evaluación de suplementos alimenticios para el Programa de Educación, Salud y Alimentación. Salud Publica Mex 1999;41:153-162.

20. Rivera J, Sotres D, Habicht J-P, Shamah T, Villalpando S. Impact of the Mexican Program for Education, Health and Nutrition (Progresa) on rates of growth and anemia in infants and young children. A randomized effectiveness study. JAMA 2004;29I(2I):2563-2570. 